\section{O Sistema Único de Saúde e as ações do enfermeiro em uma instituição hospitalar brasileira ${ }^{1}$}

\author{
Marcia Regina Antonietto \\ da Costa Melo, ${ }^{2}$ Neide Fávero, ${ }^{2}$ \\ Yolanda Dora Martinez Évora ${ }^{2}$ \\ e Janete Rodrigues da Silva Nakao ${ }^{2}$
}

\section{RESUMO}

Há uma escassez de trabalhos sobre a realidade da assistência de enfermagem nas instituições hospitalares brasileiras vinculadas ao Sistema Único de Saúde, instituído em 1988. Dessa forma, o objetivo do presente estudo foi examinar as possíveis modificações ocorridas nesta assistência desde a implantação do referido sistema. Partiu-se de entrevistas com 31 enfermeiros que atuam em um hospital universitário em Ribeirão Preto, São Paulo, admitidos pela instituição entre maio de 1980 e maio de 1987. As entrevistas, realizadas entre julho e setembro de 1995, foram examinadas a partir de análise temática e revelaram que, na opinião dos enfermeiros, os pacientes atendidos após o convênio com o Sistema Único de Saúde são mais complexos e têm melhor nível socioeconômico. Os entrevistados perceberam também um aumento na complexidade das demandas do paciente e na variedade de especialidades médicas, assim como uma diminuição no tempo de internação. Quanto às ações executadas pelo enfermeiro, $46 \%$ perceberam mudanças (por exem-

\footnotetext{
Trabalho baseado na tese de doutorado intitulada "O Sistema Único de Saúde e as ações do enfermeiro na instituição hospitalar", apresentada à Escola de Enfermagem de Ribeirão Preto da Universidade de São Paulo (EERP-USP) em agosto de 1996.

2 Universidade de São Paulo, Escola de Enfermagem de Ribeirão Preto, Departamento de Enfermagem Geral e Especializada. Correspondência e pedidos de separatas devem ser enviados a Marcia Regina A. da Costa Melo no seguinte endereço: Escola de Enfermagem de Ribeirão Preto, Universidade de São Paulo, Avenida Bandeirantes 3900, CEP 14040-902, Ribeirão Preto, SP, Brasil. Telefone: +55-16-602-3440; fax: +55-16-633-3271/630-2561. E-mail: mracmelo @glete.eerp.usp.br
}

plo, menos proximidade com o paciente). Certas dificuldades apontadas não se relacionavam com a implantação do Sistema Único de Saúde (por exemplo, o déficit quantitativo de recursos humanos). No atual momento, é essencial que o enfermeiro reflita sobre a política nacional de saúde e que se engaje na (re)construção da prática assistencial. O enfermeiro deve compreender a realidade de sua organização e exercer um processo de gerenciamento voltado para as expectativas dos clientes e dos trabalhadores em saúde.

O Sistema Único de Saúde (SUS), instituído no Brasil pela constituição federal de 1988 (1), faz parte de um processo de descentralização das ações e serviços de saúde iniciado na década de 70, que propunha a construção de uma rede única de atendimento unificado, universalizado e descentralizado do sistema público de saúde. Este processo de descentralização teve continuidade, na década de 80, com as ações integradas de saúde (AIS) e, em seguida, com o Sistema Unificado Descentralizado de Saúde (SUDS). A partir de 1990, a denominação SUDS foi substituída pela denominação SUS (2).

As AIS representaram um movimento fundamental para iniciar o processo de mudança e constituíram a estratégia de integração programática entre as instituições de saúde pública das três instâncias governamentais - federal, estadual e municipal - e os demais serviços de saúde. Embora tenham sido implantadas, as AIS não foram incorporadas à prática dos serviços de saúde.

Este impasse criou condições para a realização da $8^{\text {a }}$ Conferência Nacional de Saúde. As conferências de saúde, convocadas pelo Presidente da República, são reuniões em que a sociedade brasileira avalia a situação de saúde e propõe diretrizes para a formulação de políticas na área (3). Participam das conferências representantes de todas as instituições que atuam no setor, bem como da sociedade civil, dos grupos profissionais e dos partidos políticos (4). Partindo das conclusões da $8^{\mathrm{a}}$ Conferência Nacional de Saúde, com a concepção de um sistema único de saúde, foi criado o SUDS, cujo objetivo era consolidar o desenvolvimento qualitativo das AIS (5), tendo como diretrizes a universalização (não distinção da clientela entre contribuintes e não contribuintes do sistema) e a eqüidade do acesso aos serviços de saúde (todo indivíduo tem direito); a integralidade dos cuidados assistenciais (superação da dicotomia entre as ações preventivas e curati- 
vas); a regionalização e a integração dos serviços; a descentralização das ações de saúde; e o desenvolvimento de uma política de recursos humanos (3). Em relação a financiamento, a implantação do SUDS implicou em estratégias de repasse de recursos financeiros do nível federal para as secretarias estaduais e municipais de saúde, mediante a adesão destas secretarias ao convênio SUDS.

Em 1990, quando o SUDS passou a se chamar SUS (2), foi aprovada a lei orgânica de saúde (lei no 8080) (6), que dispõe sobre as condições para a promoção, proteção e recuperação da saúde, caracteriza o SUS e reforça os princípios já determinados desde as AIS, dentre eles universalidade, integralidade, eqüidade e hierarquização nos serviços e ações de saúde. A lei estabelece, no segundo artigo de seu primeiro parágrafo:

"Saúde é um direito fundamental do ser humano, devendo o Estado prover as condições indispensáveis ao seu pleno exercício; promover assistência a pessoas por intermédio de ações de proteção, promoção e recuperação da saúde com a realização integrada das ações assistenciais e das atividades preventivas".

O SUS agrega todos os serviços estatais (esferas federal, estadual e municipal) e os serviços privados (desde que conveniados ou contratados, com prioridade para as entidades filantrópicas). A lei orgânica da saúde deu aos municípios competência para planejar, organizar, controlar e avaliar, gerir e executar as ações e serviços públicos de saúde. Os estados prestam apoio técnico e financeiro aos municípios e executam, supletivamente, as ações e serviços de saúde.

Com o passar do tempo, as formas de financiamento do setor de saúde foram sendo modificadas significativamente, levando a um investimento cada vez menor nesta área. Este fato ampliou a importância da $9^{a}$ Conferência Nacional de Saúde em 1992, que trouxe entre seus temas centrais a municipalização da saúde. Com a municipalização, os recursos financeiros destinados à saúde são transferidos da esfera federal e estadual para o município, que gerencia sua aplicação. Esta situação leva à necessidade de repensar o papel das instituições de saúde. Os hospitais, especificamente, sem abandonar sua contribuição significativa aos cuidados primários, passaram a ser responsáveis pela assistência secundária e terciária. Neste sentido, os hospitais devem (re)definir o seu perfil assistencial e de complexidade, de maneira a oferecer tratamento adequado. Este é um dos primeiros passos no sentido de transferir à rede básica uma série de responsabilidades assistenciais e conseguir que os problemas de saúde de menor complexidade sejam ali resolvidos (7).
Desde sua implantação, o SUS tem influenciado o ambiente intra-hospitalar e, conseqüentemente, o trabalho do enfermeiro. Dessa forma, a presente investigação tem como objetivo conhecer e analisar a opinião dos enfermeiros sobre as modificações ocorridas na prestação da assistência de enfermagem em uma instituição hospitalar, após sua inserção no SUS.

\section{MATERIAIS E MÉTODOS}

O estudo foi realizado no Hospital das Clínicas de Ribeirão Preto - SP - Campus (HC), nas unidades ambulatorial e de internação clínica e cirúrgica, conveniado ao SUS desde 20 de maio de 1988 (8). O estudo consiste em uma pesquisa não experimental ex-pós-fato (9), conduzida depois que as alterações da variável independente ocorreram no curso natural dos acontecimentos. Tenta compreender as relações entre o fenômeno, como ele ocorre naturalmente, sem qualquer intervenção do pesquisador. Seu principal objetivo é determinar as relações entre as variáveis. Na pesquisa ex-pós-fato, o pesquisador não tem controle da variável independente, porque ela já aconteceu. O exame desta - o fator causador - é feito depois do fato ocorrido. Neste estudo, priorizamos o discurso de entrevistados como fonte de informação. A análise de conteúdo de Bardin (10) foi utilizada para estudar as opiniões dos entrevistados sobre as possíveis transformações ocorridas no atendimento hospitalar após a implantação do SUS.

Para garantir que os enfermeiros entrevistados estivessem familiarizados com o SUS, o estudo foi realizado dentro de um universo que englobasse todos os enfermeiros admitidos pela instituição entre maio de 1980 e maio de 1987. Assim, garantiuse que os entrevistados estivessem adaptados às suas atribuições e exercendo normalmente suas funções deste antes da adesão do HC ao SUS (em 1988) e até o momento da coleta de dados. Dos 159 enfermeiros existentes na instituição por ocasião da coleta de dados, 31 preenchiam este critério e constituíram a amostra estudada.

A entrevista foi a técnica utilizada para a coleta de dados. Para a sua realização, elaborou-se um instrumento dividido em duas partes. A primeira parte foi voltada para aspectos ligados à caracterização profissional dos sujeitos; a segunda foi destinada às questões norteadoras do estudo, com perguntas abertas e opinativas sobre possíveis transformações ocorridas na instituição hospitalar após a adesão ao SUS.

O instrumento foi submetido à validação por quatro enfermeiros da instituição, que não fizeram 
parte da amostra, e por dois enfermeiros docentes, com a finalidade de testar a objetividade, a clareza e a compreensão das questões. Após críticas e sugestões apontadas por estes, o instrumento foi modificado, com o objetivo de facilitar a compreensão pelas pessoas entrevistadas neste estudo.

Após aquiescência da Comissão de Ética Médica do HC, a coleta de dados foi realizada pelos autores no período de julho a setembro de 1995 . No primeiro contato, foi apresentado o projeto de pesquisa, esclarecido o seu objetivo, a técnica da coleta de dados e solicitada a colaboração para participar da investigação. Diante da aceitação, a entrevista era realizada ou agendada de acordo com a disponibilidade do entrevistado.

As entrevistas foram integralmente transcritas para um computador, tomando-se o cuidado de manter os termos utilizados pelos informantes. Após uma leitura flutuante das informações obtidas, optamos por organizar os dados com a ajuda do programa The Ethnograph (11). As respostas foram agrupadas de acordo com as questões norteadoras do estudo (10):

1) Você percebeu mudanças no Hospital nos últimos anos? Em caso afirmativo, quais?

2) De onde e porquê surgiram estas mudanças?

3) Após a implantação do SUS nesta instituição, em sua opinião, ocorreram transformações nas ações do enfermeiro?

a) Em caso afirmativo, o que fazia antes? $\mathrm{E}$ agora?

b) Qual o significado dessas transformações para você?

4) Houve mudanças em relação ao tipo de paciente?

a) Em caso afirmativo, como era o paciente atendido antes? E agora?

b) Esta mudança no tipo de paciente requer maior atendimento do enfermeiro? Como?

5) Como você percebe as mudanças ocorridas no setor de saúde?

\section{RESULTADOS}

Os 31 enfermeiros que preenchiam o critério de inclusão participaram da pesquisa. Destes, $55 \%$ tiveram sua formação profissional na Escola de Enfermagem de Ribeirão Preto da Universidade de São Paulo, ou seja, na escola que utiliza o hospital em estudo para estágio (único elo de ligação entre as duas instituições); 35\% haviam se formado há 11 ou 12 anos, enquanto que $32 \%$ trabalhavam na instituição havia 10 ou 11 anos. Sessenta e oito por cento dos entrevis- tados desenvolviam suas funções na unidade de internação e $32 \%$ na unidade ambulatorial. Dos entrevistados, $42 \%$ ocupavam cargos de chefia.

Quando os enfermeiros foram estimulados a relacionar que tipo de paciente era atendido antes e após o convênio HC-SUS (pelas questões norteadoras $1,4 a$ e $4 b$ ), oito (26\%) disseram não ter notado mudanças. Os 23 restantes ( $74 \%)$ mencionaram que o paciente pós-SUS é mais complexo e tem melhor nível socioeconômico.

Em relação à complexidade das patologias atendidas no HC, os entrevistados perceberam um aumento na complexidade das demandas do paciente, que passou a exigir mais cuidados por parte da equipe de enfermagem, mesmo para pacientes atendidos na unidade ambulatorial. Ainda com relação à complexidade das patologias, foi percebido um aumento nas especialidades médicas para atender a esses pacientes (como por exemplo, unidade de transplante renal e de transplante de medula óssea), assim como uma diminuição no tempo de internação. Outro ponto mencionado é que o paciente tem seguido a hierarquização dos serviços de saúde até chegar ao HC (iniciam procurando serviços de saúde de menor complexidade até chegarem ao serviço de maior complexidade).

Em relação aos recursos humanos (questões norteadoras 1, 2, 3a, 3b, 4b), pelas opiniões expressadas percebe-se que a instituição tem tido dificuldade para manter seu quantitativo de pessoal, pois apresenta elevada rotatividade. Além disto, os entrevistados mencionaram que a qualidade está abaixo dos padrões de excelência pretendidos pela instituição. A redução no número de funcionários e o aumento no número de pacientes atendidos têm provocado sobrecarga de trabalho para todos, com reflexos negativos, modificando a assistência prestada ao paciente. Um fator importante e consensual, apontado pelos enfermeiros como a principal causa da evasão de pessoal de enfermagem, são os baixos salários percebidos pelos funcionários na instituição hospitalar, favorecendo sua migração para outros setores de saúde, criados pelo SUS.

Em relação à dinâmica de trabalho do setor de enfermagem (questões norteadoras 1, 2, 3a, 3b, 4a, $4 b)$, dos 31 enfermeiros $14(46 \%)$ perceberam mudanças. Os relatos obtidos levam a crer que o trabalho do enfermeiro era mais próximo ao paciente, permitindo uma assistência de enfermagem global. Hoje, os enfermeiros disseram que, embora a assistência também seja próxima, parece ser menos sistematizada, não existindo tempo para a interação com todos os pacientes da unidade; ou a assistência direciona-se ao paciente mais grave, ou o enfermeiro enfrenta acúmulo de trabalho pois precisa substituir outros elementos da equipe. Além disso, 
os enfermeiros continuam dedicando parte do seu tempo a atividades administrativas, voltadas ao gerenciamento do cuidado. As ações administrativas mais citadas foram o controle de materiais e a supervisão de pessoal, não sendo possível diferenciar se esta ação envolve atividades educativas ou de controle.

Para 17 enfermeiros (54\%), a origem das mudanças ocorridas (questões norteadoras 1, 2,5) não foi o apenas o convênio HC-SUS, mas também a falta de pessoal. Para estes enfermeiros, outros fatores podem estar favorecendo a ocorrência de mudanças dentro da instituição. Dentre eles é possível citar as mudanças ocorridas na política governamental e institucional, a dificuldade financeira do país e da sociedade e a crise do setor saúde. Não houve consenso, entre os enfermeiros, quanto a modificações no tipo de ação por eles realizadas (assistenciais ou administrativas). Porém houve manifestação de preocupação com a assistência de enfermagem e a perda do espaço profissional.

\section{DISCUSSÃO}

A figura central dos serviços de saúde é o paciente. Quando do atendimento hospitalar, cabe ao enfermeiro a responsabilidade de gerenciar a assistência durante a hospitalização do paciente. Desta forma, a maior complexidade apresentada pelo paciente, e percebida pelos enfermeiros, vem ao encontro dos pressupostos do SUS para uma instituição no nível terciário de assistência, ou seja, que se destina a receber todo e qualquer tipo de caso, por mais complexo que ele seja. Assim sendo, o HC tem implementado especialidades médicas para suprir as necessidades da população, como, por exemplo, unidades de cirurgia de epilepsia e de transplante de medula óssea.

Há evidências da necessidade de maior integração entre as unidades de saúde, possibilitando o encaminhamento de pacientes para unidades de maior complexidade, onde os pacientes são tratados e posteriormente reencaminhados às unidades de origem para acompanhamento, estabelecendo-se, assim, a contra-referência (12). No caso do HC, é possível dizer que os princípios de regionalização, hierarquização e universalização, previstos pelo SUS, estão sendo considerados. Devido a estes princípios, a mudança em relação à complexidade e à gravidade do paciente, assim como dos que procuram pelas especialidades médicas, era esperada.

Em relação ao nível socioeconômico, anteriormente o atendimento no $\mathrm{HC}$ era voltado para as camadas sociais de mais baixa renda. A partir de 1985, com a proposta das AIS, houve eqüidade de acesso, levando a uma diversidade das camadas sociais atendidas. Além disso, em função da maior politização da população e da divulgação feita sobre o atual sistema de saúde, esperava-se que o paciente do SUS fosse mais exigente, fato este confirmado pelos enfermeiros.

Apesar de alguns enfermeiros perceberem as mudanças ocorridas no HC, após a implementação do SUS, não conseguem identificar, de maneira clara, a relação dessas mudanças com a nova proposta de organização do sistema.

Concluímos que, para o enfermeiro do $\mathrm{HC}$, o paciente atual está diferente: geralmente é portador de patologias mais complexas ou de doenças crônicas em estágio mais avançado, com restabelecimento mais difícil e maior grau de dependência da enfermagem; as internações têm se dado por períodos menores, sucedidas das reinternações, voltadas para o tratamento da patologia, ainda com foco no modelo clínico individual; o paciente está mais esclarecido e exigente quanto à sua assistência. Um número maior de pacientes têm procurado o $\mathrm{HC}$ e os princípios da regionalização, da hierarquização e da universalização estão sendo considerados pela instituição. Por estes dados, é possível inferir que as mudanças no tipo e número da clientela atendida devem-se ao convênio HC-SUS.

Em relação às mudanças para o pessoal de enfermagem, desde o início da reforma sanitária esteve presente a preocupação com os recursos humanos. A reforma sanitária propunha uma política que contemplasse remuneração condigna, capacitação permanente, estabilidade no emprego, dentre outros, mas a instituição em foco é um hospital de ensino conveniado ao SUS somente para o atendimento terciário, estando, portanto, fora desta política. Rodrigues Filho (13) lembra que os valores da classe trabalhadora estão mudando em todo o mundo, sendo que o salário tem afetado bastante a lealdade do trabalhador.

Como alternativa para tentar solucionar o déficit de funcionários, a instituição vem realizando vários concursos; mas os baixos salários oferecidos não os tornam muito atraentes aos profissionais da área.

É real a evasão de funcionários do HC para as unidades municipais de serviços de saúde. Houve um aumento de unidades básicas e distritais com a municipalização da saúde proposta pelo SUS. O recrutamento de pessoal de enfermagem para estes locais ocasionou a evasão nas várias instituições locais de saúde, principalmente hospitalares, pois os salários oferecidos são melhores. Esta competitividade dificulta a permanência de profissionais especializados e competentes na instituição.

A complexidade das atividades desenvolvidas no atendimento de enfermagem e as necessidades apresentadas pelos pacientes após a implementação 
do SUS acarretam a exigência de um quadro de pessoal quantitativamente e qualitativamente superior. Além da falta de pessoal de enfermagem, o enfermeiro realiza uma função gerencial ainda permeada pela incerteza, incompreensão e desentendimento. A este respeito, Fávero (14) relata que a administração exercida pelos enfermeiros nos hospitais tem sido passiva, obediente à instituição e voltada para a execução de rotinas, ao invés de privilegiar a criatividade, a inovação e a participação, assim como a proximidade do trabalhador e do cliente. Talvez seja necessário repensar o gerenciamento exercido pelos enfermeiros na instituição em estudo.

É impossível negar a precariedade atual dos recursos humanos. O aumento do número de funcionários por si só não eleva a eficácia nem a eficiência do serviço. Para os enfermeiros, e concordamos com eles, os principais motivos da evasão e da não reposição do pessoal são os baixos salários e não especificamente o convênio HC-SUS. Entretanto, questionamos se os enfermeiros deste estudo não estão utilizando a justificativa da precariedade de recursos humanos para encobrir atitudes de passividade, conformismo, pouco questionamento e não envolvimento frente às questões que envolvem o sistema de saúde, a instituição e a assistência de enfermagem. Merece ser mencionado que os enfermeiros percebem uma queda de qualidade em relação à assistência prestada, relacionam esta queda com a falta de recursos humanos, mas não sugerem soluções.

Leopardi et al. (15) citam que, quando os enfermeiros falam sobre seu trabalho, mostram pelo menos duas tendências: uma que privilegia o trabalho individualizado e especializado tecnicamente e outra que privilegia uma visão coletiva, na qual o trabalho é dividido com outras categorias, determinado pela instituição e parte do processo de assistência à saúde. Além disso, aqueles autores afirmam que existe uma tendência, entre os enfermeiros, a se sentirem gratificados somente quando estão junto ao paciente ("assistência direta").

Os dados encontrados neste estudo tendem a corroborar a visão de centralidade no cliente, no cuidado individualizado, principalmente na fase anterior à adesão da instituição ao SUS. Hoje, o cuidado é ministrado de forma mais rápida, voltado para situações específicas, e os enfermeiros perceberam nitidamente a queda na qualidade deste cuidado. Em relação à assistência direta, parece existir, atualmente, uma necessidade maior de sua realização pelos enfermeiros, mas em nenhum momento eles disseram "sentir-se gratificados". Ao contrário, a idéia que perpassa é a de que estão realizando estas ações pela falta de outros elementos da equipe.

Embora os enfermeiros tenham relatado seu envolvimento com a assistência direta, percebe-se que eles têm dedicado parte de seu tempo a atividades administrativas, voltadas ao gerenciamento do cuidado de enfermagem. A nosso ver, as ações de natureza administrativa realizadas pelos enfermeiros nesta instituição têm se dado de modo a "manter" a organização da unidade, o que nem sempre é possível, tendo em vista o déficit de materiais e de funcionários, de forma que as atividades administrativas se limitam à supervisão e controle de materiais e funcionários.

É preocupante que não haja consenso entre as ações realizadas pelos enfermeiros. Parece-nos que existe uma atitude de desinteresse por parte destes profissionais em relação à melhoria das condições de trabalho, uma vez que ela é o alicerce para o desenvolvimento da prática de enfermagem. Ainda quanto à perda do espaço profissional, acreditamos que o enfermeiro deve ter competência em sua área de atuação para mantê-lo e mesmo ampliá-lo.

Para categorizar as tarefas, procedimentos e atividades desenvolvidas pelos 14 enfermeiros que fizeram referência às mudanças na dinâmica de trabalho do setor de enfermagem, de acordo com a proposta de Xavier et al. (16), as ações que predominam são as de assistência, concretamente ofertadas à clientela - ações de natureza propedêutica e terapêutica, complementares ao ato do médico e de outros profissionais, e ações de natureza terapêutica e propedêutica da enfermagem, seguidas de ações de natureza administrativa, principalmente a supervisão de pessoal e o controle.

Em nossa análise, há enfermeiros que perceberam mudanças em suas ações e identificaram como causa o convênio HC-SUS, enquanto outros não identificaram mudanças. Os dados coletados sugerem que, independentemente de ter ou não percebido mudanças em suas ações, o enfermeiro parece estar cumprindo sua jornada de trabalho de forma rotinizada, pouco envolvido com o contexto geral; suas ações permanecem fragmentadas e são mais desqualificadas do que anteriormente, voltadas principalmente à complementaridade do ato médico. Percebe-se a falta de um fio condutor para o encaminhamento do trabalho, assim como a ausência de um elemento que o coordene, o que pode ser traduzido pela falta de gerenciamento adequado. Frente a isto, é possível afirmar que a assistência de enfermagem prestada neste momento não é aquela que o modelo proposto pelo SUS necessita.

Os enfermeiros relacionaram as mudanças ocorridas na instituição com os mais diversos fatores, sem, contudo, conseguir estabelecer quais foram os fatores determinantes. Os dados obtidos sugerem que, em nível institucional, é preciso que o SUS seja entendido globalmente e que os profissionais do HC sejam envolvidos neste modelo de assistência. É pre- 
ciso que os enfermeiros conheçam, entendam, analisem e questionem não só o que se passa ao seu redor, na instituição em que trabalham, mas também o sistema de saúde como um todo, para poder propor modificações. A percepção de alguns enfermeiros sobre as mudanças ocorridas no sistema de saúde caracteriza-se por uma expectativa negativa, direcionada para o que captaram e entenderam do seu local de trabalho, ou restrita ao âmbito do município.

De acordo com os dados obtidos, podemos inferir que, depois da instituição do SUS, ocorreram mudanças no HC em relação aos pacientes, aos recursos humanos de enfermagem e aos materiais, quase sempre com perdas para todos. Com base nas respostas obtidas, verifica-se que estas transformações se devem a vários fatores, dentre os quais a política governamental de saúde, a política da própria instituição, a evasão do pessoal de enfermagem e a adesão do hospital ao SUS.

\section{CONCLUSÕES}

Apesar das conquistas ocorridas no setor de saúde, como por exemplo, o processo de municipalização e a descentralização do poder, ainda estão por ocorrer as mudanças necessárias nos locais onde a assistência à saúde é produzida e realizada, incluindo-se aí a assistência de enfermagem (17).

O modelo assistencial, conforme previsto na reforma sanitária, contempla a assistência integral à saúde e a unificação das ações curativas e preventivas. Tal modelo requer um novo perfil de trabalhadores de saúde, bem como a especificação de qual produto se quer proporcionar, como deve ser produzido e para que clientela (18). Assim, é essencial que o enfermeiro perceba e reflita sobre o atual momento da política nacional de saúde.

No contexto atual do $\mathrm{HC}$, o que fazer para integrar o paciente complexo, o número reduzido de mão-de-obra na enfermagem e o redirecionamento das ações do enfermeiro, com o intuito de qualificar a assistência de enfermagem? Este nos parece ser o real desafio para a enfermagem hospitalar: a (re)construção da prática assistencial de modo a contribuir para a integralização da assistência à saúde. Neste momento, acreditamos que o enfermeiro deva desenvolver sua capacidade de gerenciar, de compreender a realidade de sua organização e de exercer um processo de gerenciamento voltado para as expectativas dos clientes e dos trabalhadores em saúde, inclusive dos enfermeiros (14).

\section{REFERÊNCIAS}

1. Brasil. Constituição da República Federativa do Brasil, 1988. Título VIII, Capítulo II, Seção II, Artigos 196 e 198.

2. Noronha JC, Levcovitz E. AIS-SUDSSUS: os caminhos do direito à saúde. Em: Guimarães R, Tavares RAW, orgs. Saúde e sociedade no Brasil: anos 80 . Rio de Janeiro: Relume Dumará, 1994. p. 73-111.

3. Brasil. Conferência Nacional de Saúde, 9, relatório final: apresentação. Brasília, 1992.

4. Brasil. Conferência Nacional de Saúde, 8, relatório final. Brasília, 1986.

5. Brasil, Ministério da Previdência e Assistência Social, Instituto Nacional de Assistência Médica da Previdência Social. SUDS. Decreto no 94657, julho, 1987.

6. Brasil. Lei orgânica da saúde: lei no 8080, de 19 de setembro de 1990. Saúde em Debate 1990;30(4):15-20.

7. Castelar RM. Gestão hospitalar: um desafio para o hospital brasileiro. Paris: ENSP; 1995.
8. Estado de São Paulo. Decreto no 28410, de 20 de maio de 1988, e resolução CIS/SP no 49/88. Diário Oficial do Estado, São Paulo, 21 de maio de 1988:3.

9. Polit DF, Hungler BP. Nursing research principles and methods. $4^{\mathrm{a}}$ ed. Philadelphia: JB Lippincott; 1991.

10. Bardin L. Análise de conteúdo. Lisboa: Persona; 1977.

11. Seidel JV, Clark JC. The ethnograph: a user's guide. Amherst: Qualis Research Associates; 1988.

12. Junqueira LAP. Organização e prestação pública dos serviços de saúde. Cadernos FUNDAP 1987;7(13):52-56

13. Rodrigues Filho J. A crise gerencial no sistema de saúde. Rev Admin Publica 1994;28(4):65-75.

14. Fávero N. O gerenciamento do enfermeiro na assistência ao paciente hospitalizado [tese de livre-docência]. Ribeirão Preto, SP: Escola de Enfermagem de Ribeirão Preto, Universidade de São Paulo; 1996.
15. Leopardi MT, Almeira MCP, Rocha SMM. O significado da assistência de enfermagem no resultado da assistência à saúde. Rev Bras Enferm 1992;45(4): 249-258.

16. Xavier IM, Souza AMA, Araújo EC, Rodrigues Neto E, Souza EO, Briceño G, et al. Subsídios para a conceituação da assistência de enfermagem rumo à reforma sanitária. Rev Bras Enferm 1987;40(2/3):177-180.

17. Silva NF. Editorial. Rev Bras Enferm 1991;44(4):5.

18. Machado MH, Carsalade MI. Os trabalhadores de saúde e o SUS. Rev Admin Publica 1992;26(2):172-178.

Manuscrito recebido em 15 de agosto de 1997. Aceito em versão revisada em 28 de abril de 1999. 
ABSTRACT There are few studies on the delivery of nursing services in hospitals participating in the Brazilian universal public health system (Sistema Unico de Saúde), which was put in place in 1988. This study, which

The Brazilian universal public health system-Sistema Único de Saúde-and the delivery of nursing services in a Brazilian hospital examined possible changes in the delivery of these services since universal health care was implemented, was based on interviews carried out between July and September 1995 with 31 nurses working at a teaching hospital in the city of Ribeirão Preto, in the state of São Paulo. The nurses had begun working at the hospital between May 1980 and May 1987. Thematic analysis was used to assess their answers. According to the nurses, patients treated after the universal system was implemented have had more complex medical needs and a higher socioeconomic status. In addition, nurses reported an increase in the complexity of patient demands and in the variety of medical specialties offered by the hospital, as well as a decrease in the length of inpatient stays. Forty-six percent of the interviewees reported a change in the work done by nursing staff (for example, nurses have less time available for each patient). Not all of the problems the nurses mentioned are related to the public health system (understaffing is one example). It is essential that nurses examine national health policy and that they engage in the (re)construction of the practice of health care delivery. Nurses ought to understand the reality of their institutions and carry out a management process geared towards the expectations of patients and of health care workers. 